\title{
Real-time monitoring of clinic risks using an integrated RFID- FA scheme
}

\author{
Nisreen A Hussein ${ }^{1}$, Mohammed M Fayyadh² \\ ${ }^{1}$ Ministry of Education, Republic of Iraq, Babil, Iraq \\ ${ }^{2}$ Faculty of Applied Science and Technology, Tun Hussein Onn Malaysia University, Johor, Malaysia
}

\begin{tabular}{l} 
Article Info \\
\hline Article history: \\
Received Sep 12, 2020 \\
Revised Dec 20, 2020 \\
Accepted Jan 12, 2021 \\
\hline Keywords: \\
Clinical risk management \\
DBSCAN \\
Firefly algorithm \\
RFID network planning
\end{tabular}

Article Info

Article history:

Received Sep 12, 2020

Revised Dec 20, 2020

Accepted Jan 12, 2021

\section{Keywords: \\ Clinical risk management \\ Firefly algorithm \\ RFID network planning}

\begin{abstract}
Patient safety is a global public health concern because of increases in the number of mistreatments due to the improper identification of patients or the improper administration of drugs. Risk in clinic management refers to the systematic process used to specify, control, and analyze organizational risks. The present article developed a new method to detect different objects automatically in real-time by monitoring and controlling the hospital workflow using radio frequency identification (RFID). The system method starts with identifying the functional area by detecting the room optical characters. Then clustering and matching the symmetrical functional area using histogram matching technique. For the monitoring process, the radio frequency network planning RNP has been used. Density-based scan algorithm (DBSCAN) was used for clustering and extracting the area, then all gathered data transferred to the firefly algorithm to track drug distribution and specify doctor and nurse locations. The simulation results observe realtime tracking and identification of people and drugs based on hospital zone designs. The results present $87 \%$ tag real-time coverage for managing and monitoring human inside the hospital. The effectiveness of this system shows that it was useful in monitoring clinic operations and effective for a hospital network solution.
\end{abstract}

This is an open access article under the CC BY-SA license.

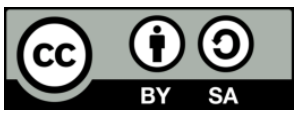

Corresponding Author:

Mohammed M. Fayyadh

Faculty of Applied Science and Technology

Tun Hussein Onn Malaysia University

Pagoh 84600 Johor, Malaysia

Email: abuzeen@gmail.com

\section{INTRODUCTION}

The management of clinical information risks is concerned with improving healthcare and service quality by preventing and controlling risk opportunities. Patient health reports are usually stored in separate systems, and healthcare providers keep a huge number of papers to comply with different regulations [1]. Most hospitals have changed the patient environment by reducing the waiting time, changing patient demographics, and creating significant practical healthcare challenges [2]. Reductions in time can be achieved through the replacement of paper-based processes with information system processes for emergency ward admissions. Hospitals with information system solutions will save time by cutting down on the requirement for doctors and nurses to fill out forms, thereby improving healthcare outcomes. Also, the potential benefits to doctors cannot be disregarded, even though the adoption of electronic health records has been slow [1]. The monitoring of patients, equipment, products, and workers in hospitals can be improved through radio-frequency identification (RFID), which represents a better solution for managing clinical risks 
[3]. The use of RFID technology can facilitate automatic patient identification processes, reduce healthcare costs, and assist in accurate and quick diagnoses and treatments [4, 5].

RFID is a wireless device that automatically detects data. This technology operates without human intervention [6]. It is used to track objects or parts to obtain specific data based on the Internet of Things (IoT) specifications. Some RFID system criteria are costs, RFID antenna collisions, network coverage, and transmitted reader power, which are calculated through mathematical models. These calculations involve reader and tag antenna specifications and RFID network planning (RNP) [7, 8].

These revolutionary features provide RFID with global and unique uses. The RFID structure provides an identification code (ID). This ID can be detected by a reader from tags, while middleware uses such IDs to monitor and track materials [9]. This technology has been used in many applications in transportation, healthcare, and other industries for tracking items, shipments, products, animals, and humans [6]. Several RFID network planning algorithms have been developed to optimize the function parameters. Gong et al. [9] proposed a novel local topology concept by applying a developed particle swarm optimization (PSO) algorithm onto a network model. Each swarm in the proposed adaptive small-world PSO (ASWPSO) algorithm interacted with its cohesive neighbors randomly and with other particles based on distance. The topology adaption mechanism was improved by assigning network specifications. Therefore, the particle swarms balanced their exploration and exploitation abilities during the swarm search process. The proposed method was tested on thirteen benchmark functions to gain high PSO robustness and efficiency [9]. Elewe et al. [10] developed a new hybrid method based on RFID and topology design. The presented method used tag density as the basis for multiobjective RFID network planning functions. The method improved cost efficiency in large and complex environments with different indoor topologies [10]. The gradient-based cuckoo search (GBCS) method and over dimensioning (OD) model achieved optimal tag coverage with a minimum number of deployed RFID readers and obtained superior results over a large area [11, 12].

This study applied a radio-frequency identification system to identify persons such as doctors, nurses, patients, and cleaners in hospital spaces, and to monitor drug movements and inventories. In particular, this project observed the functional areas in a hospital organization and detected entities based on function zones in the hospital area. The system applied the methodology of a nature-inspired algorithm to find the RFID reader positions to cover a solution that maximized the number of tags with the lowest overlapping RFID reader propagation areas $[13,14]$. The results of the nature-inspired algorithm were correlated with the tag positions in the functional hospital zone to acquire accurate information for five entities. The twodimensional plans used for the RFID network were based on the objective functions for a high RNP performance [15].

\section{SYSTEM METHOD}

The early detection of medical errors can be achieved through the use of modern information and communication systems, which improve service quality and enable prevention. Therefore, RFID systems, which allow for real-world representations to be mapped onto a virtual world, can be used for the real-time monitoring of objects. RFID systems identify objects automatically based on their signal positions and reflections using passive tags. To achieve this target, RFID systems need specified reader positions. This study encountered several problems in achieving this task. The first problem was the large area of the hospital, which presented a huge number of possibilities for the evolutionary algorithm, thereby causing weak performance and increased iteration time. The second problem was the object specifications in clinics.

The third problem was how to cluster the clinic areas functionally. All these were considered as the topological problems of the clinics. Therefore, it was necessary to organize information on the topology of the clinics to obtain high-quality results. The next section describes the system method of this study.

\subsection{Design of RFID network for healthcare}

Human medical errors in clinics cause about 42.7 adverse events each year [16]. This study used RFID to improve the quality of healthcare and to reduce human error [17]. The misidentification of people (doctors, patients, nurses, and cleaners) inside hospitals pose a risk to patient safety [18]. Also, the wrongful management of medical inventories is one cause of medication errors [19]. The early detection of defects and failures can be achieved through modern information and communication technologies, which can increase service quality and reduce healthcare costs. RFID systems, which allow the real world to be mapped onto a virtual world, can be used to monitor objects in real-time. RFID can automatically identify the people inside a clinic and objects such as drugs. This task needs reader positions with low costs. The present method correlated RFID network planning with a clinical topology network design to improve the detection and specification of objects based on the positions of doctors, nurses, patients, and drugs. This section defines the fundamentals of the clinic topology to understand the topological behavior of the tag distribution based on 
clinic design conditions. Many researchers have discussed the concept of network topology based on RFID systems. Using the methods presented in this work, a topology network design was developed based on the steps displayed.

\subsubsection{Clinic topology planning}

The first step in the method of this study was the planning of the clinic topology. In this step, a clinic layout design was drawn in .JPG form using AutoCAD. The designer had to specify and register all the functional areas based on customer requirements, as shown in Figure 1. As seen in Figure 1, the functional areas were the drug store, exam rooms, waiting area, two labs (A and B), consultant rooms, and bathrooms. This image was transferred as an input representation to the MATLAB software.

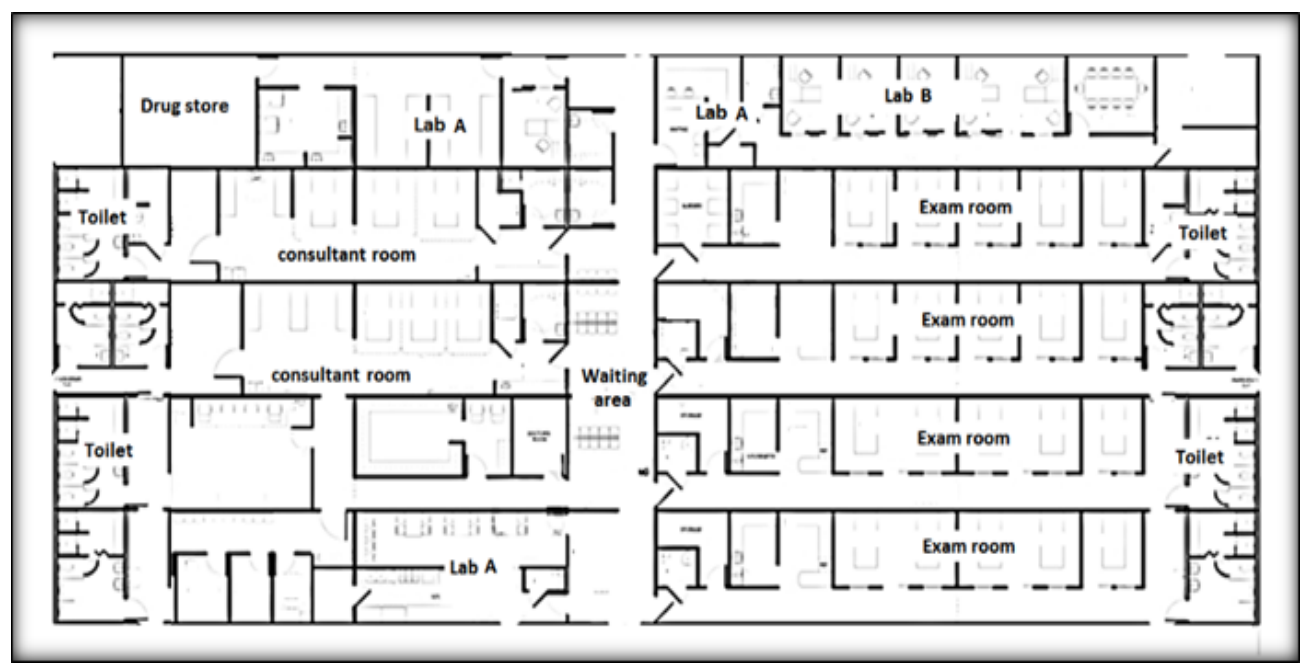

Figure 1. Clinic layout design sample

\subsubsection{Clustering process}

To cluster the clinic areas based on the functional areas, the MATLAB software was used to classify seven clinic regions based on specific optical characters written on the drawing. The process of detecting the names of the regions is shown in Figure 2. As a result of this step, the names and positions of the regions were specified, as shown in Figure 3.

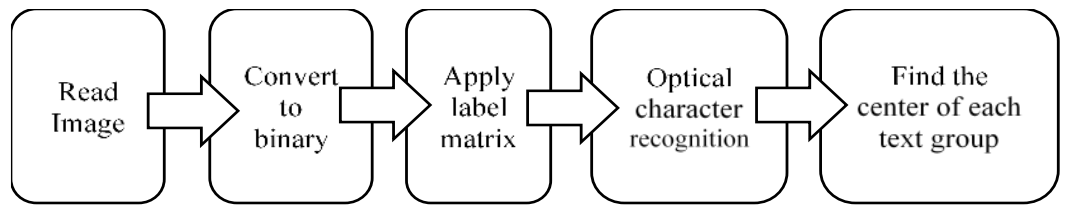

Figure 2. Clinic region detection framework

To cluster the clinic areas based on the optical characters, their characters had to be recognized and specified. The optical characters were then analyzed by histogram matching to find the symmetrical functional area. For instance, the labs in the drawing will be specified as the same function rooms [20]. The process for calculating and matching the detected optical characters starts by applying the cumulative distribution function for $F 1()$ and $F 2()$ images. Then defining the gray level of both of them $G 1$ and $G 2$. The result of the histogram will be $F 1(G 1)=F 2(G 2)$. This method is effective and flexible because it can achieve significant accuracy with less processing time and space requirements [21, 22]. The results of the proposed sequence of operations were applied to the firefly density-based algorithm [23]. This method categorizes and finds the sound that is closest to detected and specified words by analyzing the domain data of the proposed logical division. 


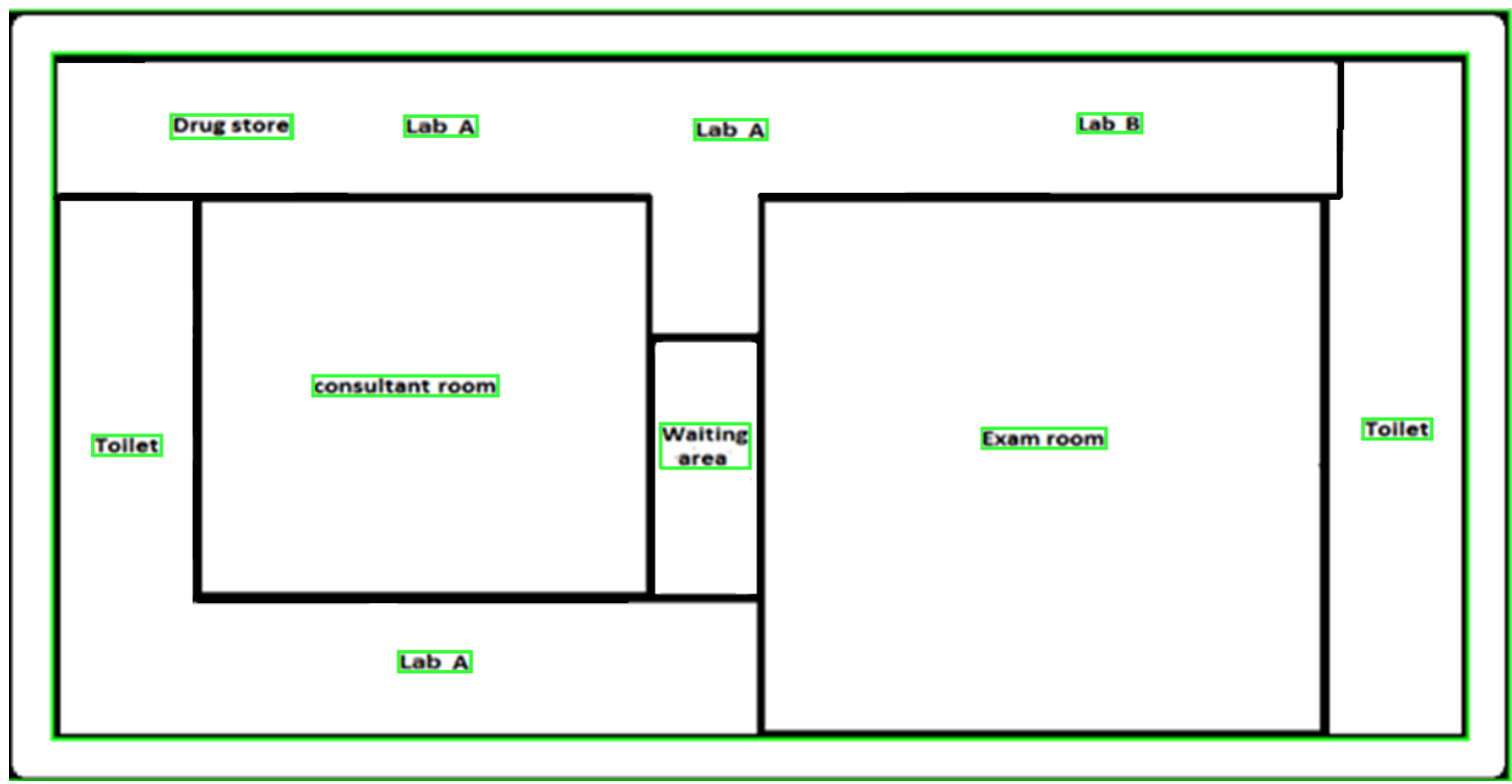

Figure 3. Region label result

In this study, a set of points in a domain were packed closely together to investigate the relationships between the pixels. Domains were defined based on the features of topological and statistical pixels. DBSCAN was used to categorize and find cluster shapes based on information from those pixels that lay close together in the labels through three definitions:

Definition 1: Specify the Eps-neighborhood of the detected label center to cluster the label character area.

Definition 2: Detect the reachable density from the label center based on direct reachable distance relationships.

Definition 3: Cluster the maximum rank of the reachable object density groups.

This technique resulted in the detection of pixel information and the grouping of data to obtain topological features such as endpoints, pixel ratios, and height to width ratios as well as statistical features such as connected components in a domain. The method worked in both the $\mathrm{x}$ - and $\mathrm{y}$-directions, as shown by the mathematical model.

$$
\begin{aligned}
& \operatorname{Cov}_{i}=\sum_{i=0}^{m} x_{i} \\
& \operatorname{Cov}_{j}=\sum_{j=0}^{n} y_{j}
\end{aligned}
$$

where $\operatorname{Cov}_{j}$ represents the covered pixels in the $x_{i}$-direction and $y_{i}$-direction. These pixels were specified based on clinic area usage. This formula clustered the clinic areas based on the room labels inserted in the drawing. Then, the clustered areas were divided into groups, based on (3), (4), (5):

$$
\begin{aligned}
& \operatorname{Sum}_{i}=\sum_{i=\min }^{i=\max } \operatorname{Cov}_{j} \\
& \operatorname{Sum}_{j}=\sum_{j=\min }^{j=\max } \operatorname{Cov}_{i} \\
& A=\iint\left(\operatorname{Cov}_{i}, \operatorname{Cov}_{j}\right) d x d y
\end{aligned}
$$

For each area, the centroid was determined to find the distances between the room centroid, detected objects, and room boundaries. The centroid represented the reference point in each specified area, as shown in (6):

$$
C_{\text {cen }}=\iint \frac{\left(\operatorname{Cov}_{i}\right)}{A} d A
$$

where $\mathrm{A}$ is the area, $\mathrm{C}_{\mathrm{cen}}$ is the centroid, the sum is the sum of the pixels, $\mathrm{n}$ is the row number, and $\mathrm{m}$ is the column number. The results of this step are shown in Figure 4. 


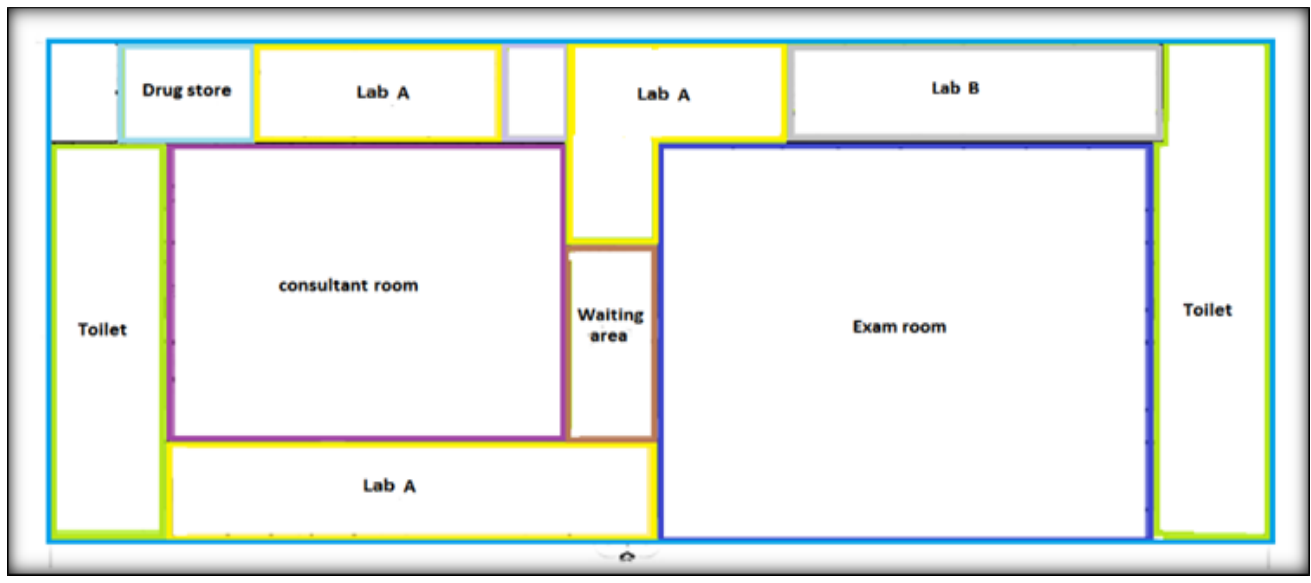

Figure 4. DBSCAN recognition results

\subsubsection{RIFD system development}

The RFID network objectives were concerned with two matters. The first was to determine the magnitudes of the optimal reader parameters, while the second was to identify the topological planning parameters of the RFID network. The optimization techniques used in this study were recent methods for detecting optimal solutions and tuning RFID network planning parameters. The values of these parameters are presented in Table 1 [24]. The objective functions of the RFID network planning can be summarized using three formulae, with the most important being optimal tag coverage (C). Optimal tag coverage expresses the ability to cover the IDs of all the tags distributed in a specified area [24].

Table 1. RFID parameters [14]

\begin{tabular}{ll}
\hline Parameters & Values \\
\hline RFID Reader system operating frequency & Uhf band: $915 \mathrm{MHz}$ \\
RFID Reader adjustable transmitting power range & {$[20 ; 33] \mathrm{dBm} 0.1$ to 2 watts } \\
Sensitivity thresholds of tags Tt & $-14 \mathrm{dBm}$ \\
Sensitivity thresholds of readers are dBm Tr & $-70 \mathrm{dBm}$ \\
RFID Reader antenna gain $(\mathrm{Gt})$ & $7.3 \mathrm{dBi}$ \\
RFID Tag antenna gain $(\mathrm{Gt})$ & $3.7 \mathrm{dBi}$ \\
Acceleration coefficients $(\mathrm{c} 1=\mathrm{c} 2)$ & 2.0 \\
Inertia weight $(\omega)$ & 0.9 to 0.4 \\
Wave length $(\lambda)$ & $0.328 \mathrm{~m}$ \\
\hline
\end{tabular}

It can be determined by the sum of the differences between the received power from the tag reflections and the actual required power transmitted from reader devices, based on the following formula:

$$
C_{\text {min }}=\sum_{i=1}^{N_{T}}\left(P_{\text {tagi }}-P_{r e q}\right)
$$

$\mathrm{P}_{\text {tagi }}=$ Actual power received from each tag

$\mathrm{P}_{\text {req }}=$ Required power threshold

$\mathrm{N}_{\mathrm{T}}=$ Number of tags in a domain

For each tag, the independent transmission power equation (Friis equation) was applied to calculate the power input at the receiving antenna based on the formulae below [24, 25]:

$$
P_{r}=\left(P_{t} \cdot G_{t} \cdot G_{r}\right) /\left(4 \pi \frac{d}{\lambda}\right)^{2}
$$

$$
\text { coverage }=\sum_{i=1}^{n t}\left[P_{\text {reader }}+G_{\text {tagi }}+G_{\text {reader }}+20 \log _{10}\left(\frac{0.026}{\sqrt{\left(x_{i}-a_{i}\right)^{2}+\left(y_{i}-b_{i}\right)^{2}}}+10\right)\right]
$$


where $\lambda$ is the wavelength $(m), P_{t}$ is the output power of the reader antenna, $P_{r}$ is the input power of the tag antenna, $d$ is the distance between the reader and the tag, $G_{t}$ is the transmitting antenna gain, and $G_{r}$ is the receiving antenna gain. Collisions in reader interface cabs were specified through the identification of the reader interrogation ranges. The interference from the powerful radiation of readers was controlled by $[24,25]$ :

$$
\text { int }=\sum_{i=1}^{N-1} \sum_{j=i+1}^{N_{\max }}\left[d_{t}\left(R_{i}, R_{j}\right)-\left(r_{i}+r_{j}\right)\right]
$$

where $N_{\text {max }}$ is the total number of readers, $R_{i}$ is the $i^{\text {th }}$ reader position, $d_{t}$ is the distance between the readers, $R_{j}$ is the $j^{\text {th }}$ reader position, $r_{j}$ is the $j^{\text {th }}$ reader's interrogation range, and $r_{i}$ is the $i^{\text {th }}$ reader's interrogation range. The interference formula was able to specify the interrogation domain of the two readers based on the total sum of the reader interrogation ranges $\left(r_{i}+r_{j}\right)$, which must be less than the distance, $d$. Also, to determine the extra readers, the following formula was used [24, 25].

$$
N_{r e q}=N_{\max }-N_{\text {extra }}
$$

The formulae presented the number of effective readers and the optimum position of each reader. The objective functions were used as the input representation for the firefly algorithm to find the optimum network planning level.

\subsubsection{Applying the firefly algorithm}

The firefly algorithm is a superior evolutionary computing model that can be used to solve complex multimodal problems. It uses nonlinear and multi-dimensional components. This algorithm is considered to be an effective optimization tool due to its high potential power [25, 26]. The leading independent parameters in this algorithm are the variations in the light intensity. This parameter follows the mathematical inversesquare law, as seen in the following formula [27]:

$$
I_{(r)}=\left(I_{0}\right) /\left(1+\gamma r^{2}\right)
$$

where $I_{(r)}$ is the light intensity, $I_{(o)}$ is the light intensity of the source, $r$ is the distance, and $\gamma$ is the light absorption coefficient. The other independent parameter is attractiveness. $\beta$ represents the attractiveness of the firefly, which is proportional to its brightness, as shown in the following (13):

$$
\beta_{(r)}=\left(\beta_{0}\right) /\left(1+\gamma r^{2}\right)
$$

where $\beta(o)$ is the attractiveness at $\mathrm{r}=0$ and the independent search space parameter. The distance between the two fireflies is determined based on the Cartesian distance:

$$
r_{i, j}=\square x_{i}-x_{j} \square=\sqrt{\sum_{k=1}^{F}\left(x_{i, k}-x_{j, k}\right)}
$$

where $\mathrm{F}$ is the number of problem parameters. When firefly $\mathrm{i}$ is attracted towards the more attractive firefly $\mathrm{j}$, its movement is defined as:

$$
x_{j}(t)=x_{i}(t)+\beta_{0} r-\gamma r_{i . j}^{2}\left(x_{j}-x_{i}\right)+\alpha(\operatorname{rand}-0.5)
$$

where $r_{i, j}$ is the distance between the fireflies, rand, is a random number uniformly distributed between 0 and 1 , and $\alpha$ is a pseudo-code randomization parameter. More details can be found in [28-30].

\section{RESULTS AND DISCUSSION}

The results of this study were obtained through the use of a firefly algorithm based on DBSCAN, which specified and clustered the clinic layout into regions before specifying and detecting the tags in each region. The tags were categorized into five types of labels for groups of peoples. In the results that were 
plotted in Figure 5, the cleaner's tags were indicated by green stars $(*)$, drugs by red stars $(*)$, patients by red plus signs $(+)$, doctors by black stars $(*)$, and nurses by blue stars $(*)$. These tag groups specified the location of each human resource or material (i.e., drugs) in the clinic. This process helped to control and monitor clinic workflow and drug movements.

The simulation results were acquired using the developed method presented in Figure 6. All the results were obtained based on the RFID network planning objective function using the specific parameters presented in the previous sections. The RFID objective function results, as well as the person recognition and drug results, were acquired. The run was done by MATLAB 2018 used 50 independent runs and 10000 iterations. The plotted results showed that the RFID readers were distributed in different areas of detected and covered tags based on different probabilities.

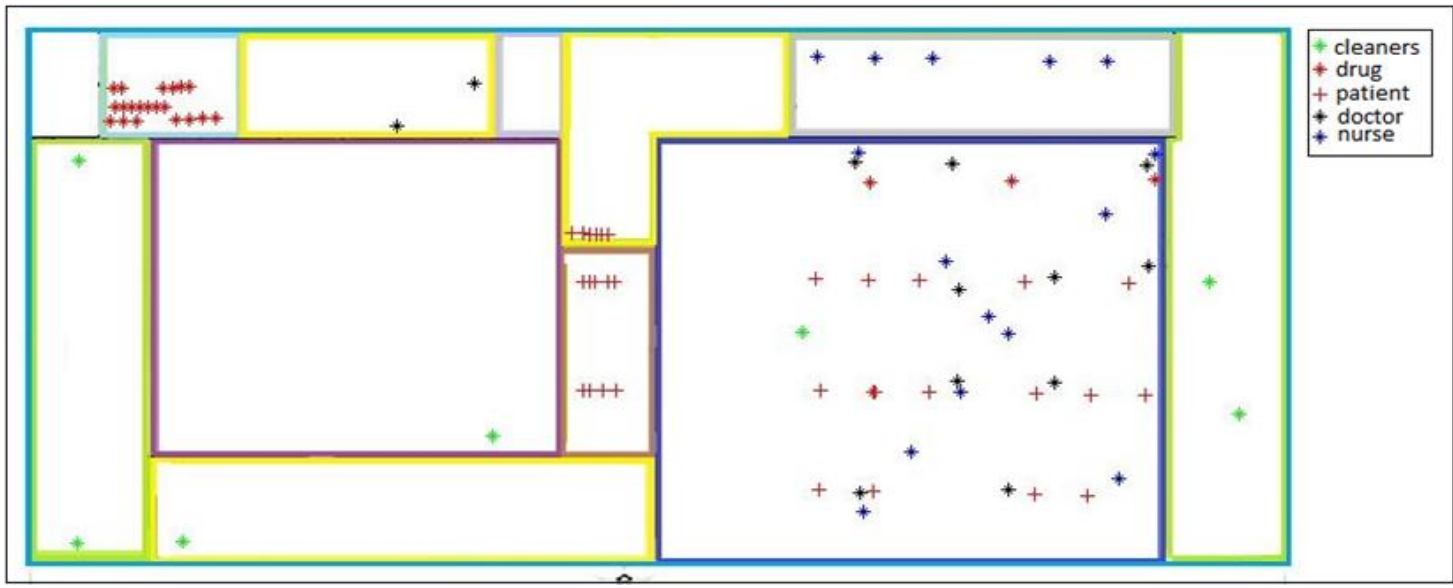

Figure 5. Tag distribution

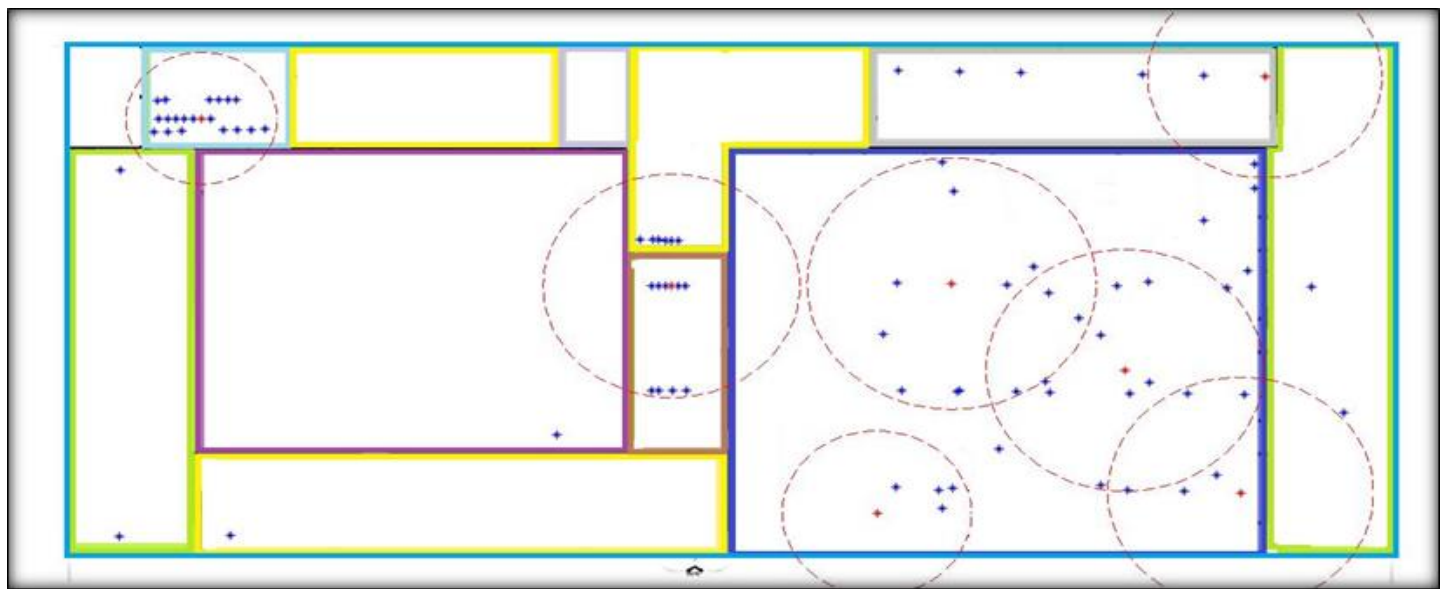

Figure 6. Simulation results

All the numerical results were arranged in Table 2 are to specify the propagation range of each reader, the (x,y) axis position of each reader, and the number of covered tags. Table 3 presents the results of the object specifications. The results showed that there were no doctors in the consultant rooms and that some drugs had been moved to the exam room. Also, there were ten nurses in the exam room and 0 nurses in the drug store. These results showed the clinic workflow and the weaknesses in the distribution of employees.

Table 2. RFID simulation results

\begin{tabular}{llllll}
\hline Algorithm & No. of readers & Interference & Tags coverage & Overlapped tags & Power transition $(\mathrm{Kw})$ \\
\hline FA & 7 & 0.2 & $87 \%$ & 3 & 22.6 \\
\hline
\end{tabular}




\begin{tabular}{llllllll}
\multicolumn{7}{c}{ Table 3. Simulation results } \\
\hline Object & Drug store & Exam room & Waiting area & Lab A & Lab B & Consultant room & Bathroom \\
\hline Doctors & 0 & 9 & 0 & 2 & 0 & 0 & 0 \\
Drugs & 50 & 4 & 0 & 0 & 0 & 0 & 0 \\
Patients & 0 & 14 & 15 & 6 & 0 & 0 & 0 \\
Nurses & 0 & 10 & 0 & 0 & 5 & 0 & 0 \\
Cleaners & 0 & 1 & 0 & 1 & 0 & 1 & 4 \\
\hline
\end{tabular}

\section{CONCLUSION}

In this paper, many specialized and advanced methods in clinic RNP network planning were addressed. A method was developed to model clinic workflow and to specify the risks in clinic operations. The workflow had to be monitored to improve the quality of healthcare services and to lower patient risk. The sequence of operations began with the detection and specification of the clinic regions to identify the positions of objects. The results showed that the positions of most of the staff members and the movements of drugs were monitored. It was shown that this system is useful and effective in monitoring clinic operations. The main contributions of this study are: Development of a novel clustering method to detect clinic rooms based on functional region; this technique provides a centroid for each room based on optical character as a reference point to manage the distances between a room and both objects (people and drugs) and room boundaries; this study combined the sequence of matching and clustering techniques with the firefly algorithm functions using RFID objective functions; the results specified the optimal reader positions based on five different tag types.

\section{REFERENCES}

[1] J. Farokhzadian, N. Dehghan Nayeri, and F. Borhani, "An RFID-based clinical information system for identification and monitoring of patients," arXiv Prepr. arXiv1503.03884, pp. 17-18, 2015. arXiv:1503.03884v1.

[2] J. Farokhzadian, N. Dehghan Nayeri, and F. Borhani, "Assessment of clinical risk management system in hospitals: an approach for quality improvement," Global Journal of Health Science, vol. 7, no. 5, p. 294, 2015. doi: $10.5539 /$ gjhs.v7n5p294.

[3] V. Di Lecce, M. Calabrese, A. Quarto, and R. Dario, "Modelling the clinical risk: RFID vs barcode," in Modeling Simulation and Optimization-Focus on Applications, IntechOpen, 2010.

[4] R. Khosla and B. Chowdhury, "Real-time RFID-based intelligent healthcare diagnosis system," in International Conference on Medical Biometrics, pp. 184-191, 2008. https://doi.org/10.1007/978-3-540-77413-6_24.

[5] M. Haddara and A. Staaby, "RFID applications and adoptions in healthcare: a review on patient safety," Procedia Comput. Sci., vol. 138, pp. 80-88, 2018. https://doi.org/10.1016/j.procs.2018.10.012.

[6] B. Khoo, P. Harris, and S. Hartman, "Security risk analysis of RFID technology." 2009.

[7] A. Azizi, "Hybrid artificial intelligence optimization technique," in Applications of Artificial Intelligence Techniques in Industry 4.0, Springer, pp. 27-47, 2009. https://doi.org/10.1007/978-981-13-2640-0_4.

[8] A. Raghib and B. A. El Majd, "Multiobjective decision aid application for RFID network planning," in MATEC Web of Conferences, vol. 200, p. 17, 2018. https://doi.org/10.1051/matecconf/201820000017.

[9] Y. Gong and J. Zhang, "Small-world particle swarm optimization with topology adaptation," In Proceedings of the $15^{\text {th }}$ Annual Conference on Genetic and Evolutionary, pp. 25-32, 2013. doi:10.1145/2463372.2463381.

[10] A. M. Elewe, K. Bin Hasnan, and A. Bin Nawawi, "Hybridized firefly algorithm for multiobjective Radio Frequency Identification (RFID) Network planning," ARPN J. Eng. Appl. Sci., vol. 12, no. 3, pp. 834-840, 2017.

[11] N. H. Talib, A. Bin Nawawi, A. M. Elewe, H. B. Abdullah, and S. Tahir, "An efficient algorithm for large-scale RFID Network Planning," in 2019 IEEE Jordan International Joint Conference on Electrical Engineering and Information Technology (JEEIT), pp. 519-524, 2019. doi:10.1109/JEEIT.2019.8717490.

[12] X. Gong, D. Plets, E. Tanghe, T. De Pessemier, L. Martens, and W. Joseph, "An efficient genetic algorithm for large-scale planning of dense and robust industrial wireless networks," Expert Syst. Appl., vol. 96, pp. 311-329, 2018. https://doi.org/10.1016/j.eswa.2017.12.011.

[13] A. M. Elewe, K. Bin Hasnan, and A. Bin Nawawi, "Review of RFID optimal tag coverage algorithms," ARPN Journal of Engineering and Applied Sciences. vol. 11, no. 12, pp. 7706-7711, 2016.

[14] A. M. Elewe, K. Bin Hasnan, and A. Bin Nawawi, "Comparative evaluation of firefly algorithm and MC-GPSO for optimal RFID Network Planning," in ICIT 2017-8th International Conference on Information Technology, Proceedings, pp. 70-74, 2017.doi:10.1109/ICITECH.2017.8079927.

[15] K. Hasnan, N. H. Talib, and A. Nawawi, "Analysis of gradient-based cuckoo search for the large scale optimal rfid network planning," in Journal of Physics: Conference Series, vol. 1150, no. 1, p. 12008, 2019. https://doi.org/10.17148/IJARCCE.2016.511119.

[16] A. K. Jha, I. Larizgoitia, C. Audera-Lopez, N. Prasopa-Plaizier, H. Waters, and D. W. Bates, "The global burden of unsafe medical care: analytic modelling of observational studies," BMJ Qual. Saf., vol. 22, no. 10, pp. 809-815, 2013. http://dx.doi.org/10.1136/bmjqs-2012-001748. 
[17] L. Hu, D. M. Ong, X. Zhu, Q. Liu, and E. Song, "Enabling RFID technology for healthcare: application, architecture, and challenges," Telecommun. Syst., vol. 58, no. 3, pp. 259-271, 2015. doi.org/10.1007/s11235-0149871-x.

[18] B. W. Podaima, M. Friesen, and R. D. McLeod, "A review of emerging smart RFID in healthcare," CMBES Proc., vol. 33, 2010.

[19] M. M. Pérez et al., "Application of RFID technology in patient tracking and medication traceability in emergency care,” J. Med. Syst., vol. 36, no. 6, pp. 3983-3993, 2012 https://doi.org/10.1007/s10916-012-9871-x.

[20] W. Dong, Z. Wang, M. Charikar, and K. Li, "Efficiently matching sets of features with random histograms," In Proceedings of the $16^{\text {th }}$ ACM International Conference on Multimedia, pp. 179-188, 2008. doi:10.1145/1459359.1459384.

[21] F. S. Mohamad, A. A. Manaf, and S. Chuprat, "Histogram matching for color detection: A preliminary study," Proc. 2010 Int. Symp. Inf. Technol.-Syst. Dev. Appl. Knowl. Soc. ITSim'10, vol. 3, pp. 1679-1684, 2010. doi:10.1109/ITSIM.2010.5561637.

[22] R. Shekhar and C. V. Jawahar, "Word image retrieval using bag of visual words," in Proceedings-10th IAPR International Workshop on Document Analysis Systems, DAS 2012, pp. 197-301, 2012. doi:10.1109/DAS.2012.96.

[23] K. Snehal Gondkar, "Face and fingerprint pattern retrieval using unique code," Glob. J. Eng. Sci. Res. Manag., vol. 4, no. 4, pp. 39-45, 2017. doi:10.5281/zenodo.556396.

[24] K. Hasnan, A. Ahmed, Badrul-Aisham, Q. Bakhsh, K. Hussain, and K. Latif, "A novel optimal RFID network planning by MC-GPSO," Indian J. Sci. Technol., vol. 8, no. 17, pp. 1-7, 2015. doi:10.17485/ijst/2015/v8i17/76221.

[25] A. A. Soomro, "Optimization of supply chain management by simulation based RFID with XBEE network," P.hD Thesis, Universiti Tun Hussein Onn Malaysia, 2015.

[26] X. S. Yang and X. He, "Firefly algorithm: recent advances and applications," Int. J. Swarm Intell., vol. 1, no. 1, pp. 36-50, 2013. doi:10.1504/IJSI.2013.055801.

[27] S. K. Pal, C. S. Rai, and A. P. Singh, "Comparative study of firefly algorithm and particle swarm ptimization for noisy nonlinear optimization problems," Int. J. Intelligent Systems and Applications, vol. 4, no. 10, p. 50, 2012. doi:10.5815/ijisa.2012.10.06.

[28] N. Bacanin, M. Tuba, and R. Jovanovic, "Hierarchical multiobjective RFID network planning using firefly algorithm," in 2015 International Conference on Information and Communication Technology Research, ICTRC 2015, 2015. doi:10.1109/ICTRC.2015.7156477. Sci., pp. 282-285, 2009. doi: 10.1007/978-3-642-04944-6_1.

[29] X. S. Yang, "Firefly algorithms for multimodal optimization, in: Stochastic Algorithms: Foundations and Applicaions," Lect. Notes Comput. Sci., pp. 169-178, 2009. doi: https://doi.org/10.1007/978-3-642-04944-6_1.

[30] X. S. Yang, "Firefly algorithm, Levy flights and global optimization," in Research and Development in Intelligent Systems XXVI: Incorporating Applications and Innovations in Intelligent Systems XVII, pp. 209-218, 2010. doi: 10.1007/978-1-84882-983-1_15. 